\title{
Laudatio: Dra. Christina Maslach, Comprendiendo el Burnout ${ }^{1}$
}

\author{
Víctor Olivares Faúndez
}

Es muy difícil poder sintetizar en estas pocas páginas el aporte de la profesora Maslach al desarrollo de la disciplina psicológica mundial, pues su fructífero trabajo tiene múltiples ribetes en la comprensión de las variables psicosociales en el comportamiento humano. Trabajo que ha abierto una nueva perspectiva universal sobre el desarrollo de los complejos fenómenos psicosociales, que tienen la poderosa capacidad de deteriorar a las personas en sus diversos contextos de desenvolvimiento, especialmente en el ámbito laboral.

En este sentido no podemos hacer referencia a la gran figura de la profesora Maslach sin asociarla al concepto de "burnout" [término anglosajón, que en lengua española hace referencia a "quemarse por el trabajo"], fenómeno que si bien tuvo como precursor al médico psiquiatra Freudenberger ${ }^{1}$, es la doctora Maslach su principal investigadora, otorgándole a este desconocido síndrome el posicionamiento mundial como fenómeno que, insidiosa y progresivamente, afecta de manera determinante la calidad de vida de los seres humanos, visión transcultural y global.

El burnout nace en EE.UU. a mediados de los años setenta como una forma de describir las reacciones adversas que se producian en el trabajo de los servicios sociales, siendo la psicóloga social Maslach $^{2}$ quien, sustancialmente, comenzó a reflexionar sobre este fenómeno, utilizando el término "burnout" para describir un nuevo síndrome clínico caracterizado por el agotamiento que se observaba entre los profesionales de la salud mental. De esta forma, Maslach dio cuenta en sus estudios de la relevancia en considerar los aspectos emocionales que operaban en el burnout, aduciendo que la tensión laboral es una variable interviniente significativa en el proceso de estrés y agotamiento psíquico, y que adecuadas estrategias de afrontamiento tenían implicaciones importantes para los individuos en cuanto a su identidad profesional y conductas laborales. ${ }^{3}$

Desde su aparición, el burnout se ha definido de muchas maneras. Sin embargo, la más repetida definición que presenta este conocido fenómeno psicológico es la planteada por Maslach y Jackson $^{4}$, quienes lo definen como un sindrome caracterizado por agotamiento emocional, despersonalización y baja realización personal en el trabajo, que puede ocurrir entre individuos cuyas tareas diarias se circunscriben al servicio de personas. El burnout

\footnotetext{
1 Laudatio leído en la ceremonia de entrega del Doctorado Honoris Causa a doctora Christina Maslach por parte de la Universidad de Valparaíso el 20 de octubre de 2016.
}

Correspondencia / Correspondence:

Victor Olivares Faúndez

School of Psychology, University of Santiago, Chile (USACH).

Avenida Ecuador \# 3650, $3^{\circ}$ Piso, Santiago, Chile.

e-mail: victor.olivares.f@usach.cl parece ser una respuesta a los estresores interpersonales en el ambiente laboral, donde el exceso de contacto con los individuos receptores de servicios produce cambios negativos en las actitudes y en las conductas hacia estas personas. ${ }^{5}$ En la actualidad, Maslach define al burnout como una respuesta prolongada al estrés en el trabajo, un sindrome psicológico que nace de la tensión crónica producto de la interacción conflictiva entre el trabajador y su empleo.

De esta forma, Maslach posiciona a este importante fenómeno como una problemática particularmente real, donde existen respuestas disfuncionales del individuo en contextos laborales caracterizados por fuertes niveles de presión y tensión laboral. Un problema sociolaboral consecuencia del estrés laboral crónico, una reacción nociva ante una tensión ocupacional progresiva e intensa.

Tal es la influencia de los postulados sobre el burnout expuestos por la doctora Maslach que se sustentan de su impecable trayectoria científica, que la abundante literatura existente sobre este fenómeno en el orbe, de forma casi genérica, se sostiene en sus fundamentales premisas. Prueba de ello son las búsquedas de importantes revisiones sobre el tema, como la que abarcó el período $1990-2002^{6}$, la cual identificó 2.138 elementos distintos asociados al burnout, y la realizada en Google Scholar, bajo el término clave burnout, que produjo más de 260.000 entradas $^{7}$, revisiones que en más de un $90 \%$ hacian referencia a la definición del sindrome postulada por la Doctora Maslach y/o a su emblemático instrumento de medición, el Maslach Burnout Inventory (MBI). Tendencia que en años recientes, aún continúa. $^{8-10}$

La investigación de la profesora Maslach acerca de esta patología, en estas tres últimas décadas, la ha llevado a cobrar un gran protagonismo como fenómeno social y científico ${ }^{11}$, ya que un hecho notable en el desarrollo del burnout es que surge como un problema social identificado por los trabajadores, mucho antes de que se convirtiera en un foco de estudio sistemático por los investigadores. Estos orígenes populares no académicos del sindrome contribuyeron a etiquetar al fenómeno de forma imprecisa, configurándose un vasto volumen de perspectivas y postulados emergentes que dificultó, en un primer momento, la claridad conceptual del sindrome, cuestión fundamental que la doctora Maslach desentrañó.

Para la profesora Maslach el burnout es una enfermedad laboral emergente, que con frecuencia se entiende que es exclusiva de profesionales de ayuda o de servicios quizás porque, al contemplar la literatura sobre este fenómeno, se comprueba que la mayoría de los estudios realizados han empleado muestras de profesionales de la educación y de la salud. Prueba de esto es el clásico estudio de Lee y Ashforth $^{12}$ en los años noventa, donde aproximadamente el 80\% de las investigaciones consideradas en el meta-análisis, se realizaron en personal de servicios humanos, y la mayor parte de las investigaciones restantes se habian realizado en supervisores y encargados de tales servicios. Esto, probablemente, es la causa de que la difusión del burnout sea mayor en este tipo de colectivos profesionales, y no en 
otros de distinta naturaleza, aspecto que hoy en día se replica en las investigaciones sobre el tema.

Así, al avanzar la década de los años noventa el concepto del burnout se fue ampliando, incluyéndose dentro de sus límites de acción ocupaciones distintas a la de los servicios humanos ${ }^{13}$, delimitando su alcance a todo profesional que trabaja con clientes de trato directo, y no sólo en personal de ayuda o del sector servicios. Esta generalidad del espectro de acción del burnout es para algunos autores aun más amplio, ya que entienden que el síndrome se puede presentar en aquellos grupos ocupacionales que trabajan con "cosas" (por ejemplo, en trabajadores del sector cerámico) y con "datos" (como usuarios de tecnologías y teletrabajadores) ${ }^{14}$, ideas que reafirman los postulados planteados por la profesora Maslach, que señalan que este fenómeno se puede desarrollar en cualquier profesión cuyo trabajo no conlleve necesariamente el contacto directo con personas ${ }^{3}$, siendo potencialmente un síndrome que se puede desarrollar en todos los tipos de actividad laboral ${ }^{15}$, situación que universaliza las ideas de esta importante investigadora, y abren un nicho importante de desarrollo sobre el tema en el espectro mundial.

A casi un cuarto de siglo de investigación empírica sobre el burnout, centrada en los trabajos de la profesora Maslach, de acuerdo a las líneas teóricas que han tratado de determinar los límites conceptuales de este fenómeno, se pueden identificar dos perspectivas: la clínica y la psicosocial. En el lado clínico, el foco de atención está centrado en los sintomas y sus aplicaciones en el contexto de la salud mental (estado) ${ }^{16,17}$, y en el lado psicosocial el foco de atención está en el desarrollo de la relación entre el medio laboral y las características humanas de los individuos partícipes de esos contextos de servicio (proceso) ${ }^{18}$, perspectiva original de la profesora Maslach, que sustenta el devenir de la investigación actual del tema.

Dentro de este postulado, se plantea que la denominación "estar quemado" sugiere una comprensión errónea del síndrome, ya que pudiese ser entendido como el término de un proceso o su consecuencia final, configurándose una concepción estática, que no obedece a la real naturaleza del fenómeno.

Según los planteamientos de Maslach, el burnout es una respuesta progresiva e insidiosa, que evoluciona como parte de un proceso mayor de estrés laboral, preferentemente en profesionales de servicios, pero no exclusivamente.

El burnout a menudo ha sido conceptualizado en el marco de la investigación del estrés ${ }^{19,20}$, por lo que su definición se sustenta en la ambigüedad que posee este concepto ${ }^{21}$, término general, donde frecuentemente las causas y las consecuencias se confunden. ${ }^{22}$

Por ello, desde sus inicios el burnout ha sido un concepto difícil de interpretar, no existiendo hasta nuestros dias una definición estándar de él que estructure una delimitación conceptual clara y precisa. Hoy en día se ha empleado para significar ideas muy distintas y, por lo tanto, no existe una buena base para una comunicación constructiva que dimensione la problemática real, dé luces a un diagnóstico unánimemente acertado y establezca posibles soluciones e intervenciones certeras, que sean consensuadas por la comunidad científica internacional. Cuestión que nos habla y pone en valor el sostenido trabajo realizado por la profesora Maslach a lo largo de estos años, ya que abrió una nueva forma de comprender estos complejos fenómenos desde una perspectiva transcultural y global.

Sin embargo, hoy en día existe un consenso amplio de opiniones sobre las cuales se entiende que el burnout es una respuesta del individuo al estrés laboral crónico. ${ }^{3}$ Una experiencia subjetiva interna que agrupa sentimientos y actitudes, y que tiene un cariz negativo para el sujeto dado que implica alteraciones, problemas, y disfunciones psicofisiológicas como consecuencias nocivas para la persona y para la organización.

\section{DIFERENCIACIÓN CONCEPTUAL DEL BURNOUT}

Variados han sido los conceptos que a través del tiempo se han relacionado con el burnout, lo cual ha llevado confusión respecto de su adecuada delimitación y alcances verdaderos.

Dada la gran complejidad del sindrome, frecuentemente se han vinculado a éste, de forma directa o solapada, conceptos como tedio $^{23}$, depresión ${ }^{24,25}$, estrés laboral ${ }^{3}$ e insatisfacción laboral ${ }^{18}$, entre otras posibles respuestas al estrés (tensión, ansiedad, conflicto, fatiga, presión, nerviosismo, aburrimiento, agotamiento físico y psíquico, alienación, cansancio, neurosis existencial, indefensión, desencanto, etc.).

Muchos han sido los autores que han relacionado tedio con el burnout, situación que nos remonta a los estudios clásicos de Pines, Aronson y $\mathrm{Kafry}^{26}$ (p. 15) y de Pines y Aronson ${ }^{23}$, donde en un comienzo definen tedio como una experiencia general de agotamiento físico, emocional y de aptitudes, definición no lejana de la planteada por estos mismos autores para el burnout. ${ }^{27}$ Posteriormente, Pines et al. abandonan esta equiparidad conceptual entre tedio y burnout, ampliando su concepción de este último, concluyendo que el burnout es un fenómeno de mayor amplitud que el tedio, pudiendo ser éste sólo un síntoma más del burnout, entendiéndolo más tarde como un predictor del sindrome ${ }^{28}$, o una medida de agotamiento. ${ }^{29}$

Quizás uno de los conceptos más controvertidos a la hora de diferenciarlo del burnout sea el de depresión, ya que ambos conceptos, junto con poseer ciertas similitudes como fatiga, dificultades para concentrarse y relajarse en el trabajo ${ }^{27}$, tienen a su vez un cierto solapamiento respecto de los sentimientos de agotamiento emocional asociados en éstos (véase los estudios clásicos de Glass y McKnight). ${ }^{30}$

La relación entre depresión y burnout fue establecida por estudios empíricos realizados usando instrumentos como el MBI y distintas medidas de depresión. ${ }^{31}$ Aquellas investigaciones concluyeron que el burnout se desarrollaria más propiamente en contextos del trabajo y situaciones específicas de éste, no interfiriendo necesariamente en otras áreas de funcionamiento de una persona, mientras que la depresión se enmarcaría dentro de contextos muchos más generales, afectando las actividades y funcionamiento global de los individuos. $^{3}$

Muchos estudios postulan que la depresión y el burnout son dos fenómenos diferentes, y que la relación estrecha existente entre ambos está basada en algunas similitudes sintomatológicas. ${ }^{27}$

Cuando intentamos relacionar insatisfacción laboral con el burnout, nos vemos ante dos conceptos muy estrechamente ligados, pues son dos experiencias psicológicas internas y de índole negativas, pero distintas en su delimitación, siendo la naturaleza que los une aún materia de especulación en el campo científico. ${ }^{3}$ Situación ${ }^{4}$ corroborada por estudios que han destacado que el burnout causa bajos niveles de satisfacción laboral y no viceversa, concluyéndose, por tanto, que la insatisfacción es una consecuencia más del sindrome. ${ }^{18}$ Asimismo, se ha encontrado en diferentes estudios que, aunque la insatisfacción laboral aparece como un componente más en el burnout, no necesariamente la insatisfacción tiene que estar 
ligada a elevados niveles de agotamiento emocional, pudiendo coexistir éste con una buena satisfacción laboral. ${ }^{32}$

Tal vez el estrés laboral sea el concepto que más confusión conlleve cuando tratamos de delimitar el burnout. Desde la definición clásica de Selye ${ }^{33}$, que señalaba que el estrés era una respuesta no específica del organismo resultante de una demanda sobre el mismo, teniendo efectos mentales o somáticos, el concepto ha presentado múltiples problemas en su definición y significado, ya que ha sido entendido como respuesta, estímulo e interacción, no llegándose hasta nuestros dias a un acuerdo sobre éste (véase Hart y Cooper). ${ }^{34}$

Desde una aproximación transaccional ${ }^{35}$, el estrés laboral es entendido como una interacción entre la persona y el entorno, en que la respuesta del estrés (activación fisiológica y cognitiva excepcional) dispone al organismo para hacer frente a una demanda del medio (externa o interna) que excede los recursos del individuo, originando un cambio en su estado habitual de bienestar de naturaleza compleja.

$\mathrm{Al}$ parecer la investigación nos señala que el burnout se configuraría como una de las variadas respuestas del estrés laboral crónico ${ }^{36}$, existiendo un solapamiento continuo entre las respuestas (psicológicas, fisiológicas y conductuales) y lo que son sus consecuencias. En este sentido Evans y Fischer ${ }^{37}$ consideran que el burnout es una respuesta al estrés laboral, que se manifiesta en individuos cuyo trabajo se caracteriza por generar relaciones de ayuda a otras personas, situación que daría sentido y comprensión a la aparición de la despersonalización como uno de los elementos definitorios del burnout, y no así del estrés laboral.

Un elemento más a favor de la delimitación conceptual del burnout respecto del concepto de estrés laboral lo constituye la posibilidad de incluir el proceso de desarrollo del propio síndrome en el episodio más amplio del estrés laboral, donde el burnout es una etapa final de un curso crónico. ${ }^{38}$

Según la doctora Maslach ${ }^{11}$, un aspecto diferenciador entre estos dos conceptos es el hecho de que el estrés puede ser entendido como un proceso que tiene efectos positivos y negativos para el individuo, mientras que el burnout sólo tendría efectos de índole negativo.

\section{Modelo tridimensional del burnout de Maslach}

Existe una gran dificultad en identificar los síntomas del burnout, dada la gran cantidad de fenómenos asociados al trastorno que destacan las investigaciones sobre el tema, y señalan hasta más de 100 síntomas asociados al síndrome, lo cual lleva a la confusión. No obstante, podemos identificar grupos caracterizados por influir negativamente en los afectos y las emociones (como agotamiento emocional, irritabilidad, odio, etc.), en las actitudes (como cinismo, despersonalización, apatía, hostilidad, etc.), en las cogniciones (como baja ilusión por el trabajo, dificultad de concentración, baja realización personal en el trabajo, etc.), en las conductas (como comportamiento suspicaz y paranoide, aislamiento, consumo de tranquilizantes, rigidez, quejas constantes, absentismo, etc.), y en el sistema fisiológico del individuo (como cansancio, insomnio, úlcera de estómago, dolor de cabeza, fatiga, hipertensión, etc.).

Maslach y Jackson ${ }^{39}$ entienden que el burnout es una respuesta al estrés laboral crónico, conformado por actitudes y sentimientos negativos hacia las personas con las que se trabaja, y hacia el propio rol profesional. Siendo un sindrome caracterizado por la vivencia de encontrarse emocionalmente agotado, la doctora Maslach concluye que el burnout se configura como "un síndrome tridimensional caracterizado por agotamiento emocional, desper- sonalización y reducida realización personal”. Más tarde, Maslach at al., ${ }^{3}$ añaden que el burnout es una respuesta a los estresores interpersonales crónicos que se desarrollan en el trabajo, donde los sintomas dominantes de esta respuesta están caracterizados por la presencia de un agotamiento abrumador, sensaciones de cinismo en el trabajo, y un sentido de ineficacia y carencia de la realización personal.

Agotamiento emocional es el elemento clave del síndrome, y se refiere a que los individuos presentan sentimientos de estar emocionalmente agotados y sobrepasados en cuanto a sus recursos emocionales. Despersonalización implica actitudes negativas, cínicas e impersonales, generándose sentimientos demasiado distantes hacia otras personas. Por último, la falta de realización personal en el trabajo se refiere a la disminución de los sentimientos de competencia y éxito en el trabajo, así como una tendencia a evaluarse negativamente a sí mismo, particularmente en el trabajo con otras personas. $^{3,39}$

Esta definición sintomática, propuesta por la profesora Maslach, ha sido utilizada de forma casi unánime por los distintos estudiosos del tema, siendo sin duda la conceptualización más aceptada del burnout en el concierto investigativo del orbe. Prueba de aquello es el consenso de las definiciones posteriores, centradas en una, algunas o todas las dimensiones sintomáticas originales propuestas por estas dos autoras. ${ }^{40}$

\section{El "Maslach Burnout Inventory" (MBI) de Maslach y Jackson $(1981,1986)$}

El Maslach Burnout Inventory (MBI) de Maslach y Jackson ${ }^{4,41}$ es un instrumento conformado por 22 ítems, que valoran en una escala de frecuencia de siete grados, los tres sintomas o dimensiones que definen el burnout que, según definen estas autoras, son Agotamiento Emocional (en adelante AE), Despersonalización (en adelante DP) y baja Realización Personal en el Trabajo (en adelante RPT).

En su versión original ${ }^{4}$, la escala para valorar los items constaba de dos formas, una de frecuencia y otra de intensidad, donde el individuo debía contestar a cada ítem a partir de preguntas relativas a sentimientos y pensamientos relacionados con el ámbito de trabajo y su desempeño habitual en éste. Sin embargo, en la versión de 1986 no se presenta la forma de intensidad porque Maslach y Jackson consideraron que ambas formas se solapaban, disponiendo como correlación la más alta entre ambas dimensiones de evaluación ( $\mathrm{r}=$ 0,73 con una media de $r=0,56$ ). La razón de mantener el formato de frecuencia es por su similitud con el utilizado en otras medidas de tipo autoinforme, de actitudes y sentimientos. ${ }^{6}$

Este inventario se divide en tres subescalas que se ajustan a tres factores ortogonales cada una, denominados: agotamiento emocional (Emocional exhaustion), (9 items), despersonalización (Depersonalization), (5 items) y realización personal en el trabajo (Personal accomplishment), (8 items), obteniéndose en ellas una puntuación estimada como baja, media o alta, dependiendo de las diversas puntuaciones de corte determinadas por las autoras para cada profesión en específico, tomando como criterio de corte el percentil 33 y el 66.

Para que el MBI tuviera forma hubo de pasar ocho años de estudio y análisis. Originalmente el MBI en su versión de 1981 estaba conformado por 25 items distribuidos en cuatro escalas, las tres anteriormente expuestas y otra denominada "Implicación con el trabajo", siendo ésta eliminada en la nueva versión de 1986, quedándose definitivamente establecido en 22 el número de ítems. 
En la última edición del manual ${ }^{42}$ se presentan tres versiones del MBI. En primer lugar, encontramos el MBI-Human Services Survey (MBI-HSS), dirigido a los profesionales de servicios humanos. Este instrumento es la versión clásica del MBI. ${ }^{4}$ Está constituido por 22 items que se distribuyen en 3 escalas para evaluar la frecuencia con que los profesionales perciben baja realización personal en el trabajo (tendencia a evaluarse negativamente, de manera especial con relación a la habilidad para realizar el trabajo y para relacionarse profesionalmente con las personas a las que atienden) (8 items), agotamiento emocional (no poder dar más de sí mismo en el ámbito emocional y afectivo) (9 ítems) y despersonalización (desarrollo de sentimientos y actitudes de cinismo y, en general, de carácter negativo hacia las personas destinatarias del trabajo) (5 ítems). Luego encontramos el MBI-Educators (MBI-ES), que es la versión para profesionales de la educación. Esta versión cambia la palabra paciente por alumno, reproduce la misma estructura factorial del MBI-HSS y mantiene el nombre de las escalas. Y, finalmente, el MBI-General Survey (MBI-GS) ${ }^{42}$, una nueva versión del MBI, que presenta un carácter más genérico, no exclusivo para profesionales cuyo objeto de trabajo son los servicios humanos. Aunque se mantiene la estructura tridimensional del MBI, sólo contiene 16 items y las dimensiones se denominan eficacia personal (6 items), agotamiento (5 items) y cinismo (5 items).

\section{El Maslach Burnout Inventory (MBI) y su espectro universal}

A partir del surgimiento del MBI, ha nacido una vasta investigación empírica que ha tratado de determinar la veracidad de las propiedades psicométricas del instrumento, y la búsqueda confirmatoria de la tridimensionalidad del síndrome propuesta en sus escalas, tanto en muestras de profesionales como en estudios transculturales.

A continuación se expondrán algunas de las principales fortalezas que presenta el MBI:

1. La gran aceptación internacional: el MBI se ha aceptado extensamente en todos los países de América Latina, la UE y en los EE.UU. ${ }^{3,43}$ Esto es una ventaja porque permite comparar resultados y desarrollar estrategias de prevención y tratamiento del trastorno, a la vez que impulsa el desarrollo de adaptaciones del cuestionario. $^{7}$

2. Importante apoyo empírico de la estructura factorial: diferentes estudios factoriales de carácter exploratorio han reproducido una estructura de tres factores similar a la del manual para las diferentes versiones del MBI, ya sea mediante rotación ortogonal o rotación oblicua. Así, por ejemplo, Aluja, Blanch y García ${ }^{44}$ confirmaron la estructura factorial de tres factores que explican el $43,39 \%$ de la variación (rotación ortogonal), con valores para agotamiento emocional de 19,45\%, baja realización personal en el trabajo de 15,07\%, y despersonalización de 8,86\%. Resultados similares se han encontrado en múltiples investigaciones apoyando la tridimensionalidad del sindrome. ${ }^{14,45}$ Asimismo, numerosos estudios que han utilizado análisis factorial confirmatorio recomiendan asumir una estructura de tres factores. ${ }^{46,47}$

3. Evidencia de validez concurrente: Maslach y Jackson ${ }^{41}$ obtuvieron correlaciones significativas entre diversas escalas conductuales y el MBI, utilizando como estimaciones esposas y compañeros de trabajo. Al parece, la puntuación global del MBI mide parcialmente el mismo constructo que el Tedium Measure (TM), el Staff Burnout Scale (SBS-HP) y el Meier Burnout Assessment (MBA), siendo el 25\% la varianza que estos instrumentos explican del MBI.

4. Evidencia de validez divergente: se ha evidenciado la validez divergente de la escala por las correlaciones significativas que se han encontrado entre las puntuaciones de dicha escala y las descripciones ante sus clientes, la sobrecarga objetiva de trabajo, el tiempo total de contacto directo con los pacientes y con las conductas del trabajador en el hogar, evaluadas por sus parejas, a la vez, de las bajas correlaciones con deseabilidad social y las correlaciones negativas con satisfacción en el trabajo.

En los ochenta se postulaba que el MBI se había aplicado en más del 90\% en todos los estudios empíricos del fenómeno en el mundo, postulado que vislumbra que este instrumento, sin lugar a dudas, es el más importante en la medición de este complejo fenómeno psicosocial. $^{39,41}$

\section{COMENTARIO FINAL}

En sintesis, los postulados de la doctora Maslach han abierto un nuevo espectro en el estudio de los complejos fenómenos psicosociales en el mundo, los cuales tienen la poderosa capacidad de deteriorar a las personas en sus diversos contextos de desenvolvimiento, especialmente en el ámbito laboral. Hablar de la obra científica de la profesora Maslach, sin lugar a dudas, nos remite al surgimiento del burnout, peligrosa afección que condiciona de forma fundamental la calidad de vida de las personas, que gracias a los sustanciales aportes científicos de la doctora Maslach, sabemos de sus alcances transculturales y globales.

En este sentido no podemos dejar de mencionar su emblemático instrumento de medición, el Maslach Burnout Inventory (MBI), herramienta psicométrica más aceptada en la disciplina psicológica y campos asociados, obteniendo un sostenido apoyo empírico en amplias muestras de diversas naturalezas ocupacionales del orbe. Desde esta perspectiva, el MBI es el instrumento que más ha aportado a la conceptualización del sindrome, obteniendo valores óptimos de validez y fiabilidad, lo cual ha ayudado, junto a diagnosticar ${ }^{8}$ el síndrome, a teorizar su desarrollo e intervenir en los diversos contextos organizacionales, mejorando de esta forma las condiciones de calidad de vida laboral de miles de personas en todo el mundo. 
1. Freudenberger HJ. Staff burnout. J Soc Issues. 1974;30(1):159-165.

2. Maslach, C. Burned-out. Hum Behav. 1976; 5:16-22.

3. Maslach C, Schaufeli W, Leiter M. Job burnout. Annu Rev Psychol. 2001;52:397 422.

4. Maslach C, Jackson SE. MBI: Maslach Burnout Inventory Manual. Palo Alto, CA: Consulting Psychologists Press; 1981.

5. Leiter M, Maslach $\mathrm{C}$. The impact of interpersonal environment on burnout and organizational commitment. J Organ Behav. 1988;9:297-308.

6. Boudreau R, Nakashima J. A bibliography of burnout citations, 1990-2002. Winnipeg, CA: ASAC; 2002.

7. Shirom A. Job-related burnout. In: Quick JC, Tetrick LE, editores. Handbook of occupational health psychology. Washington, DC: American Psychological Association; 2003.

8. Halbesleben JR. Sources of social support and burnout: A meta-analytic test of the conservation of resources model. J Appl Physiol. 2006; 91:1134-1145.

9. Melamed S, Shirom A, Toker S, Berliner S, Shapira I. Burnout and risk of cardiovascular disease: Evidence, possible causal paths, and promising research directions. Psychol Bull. 2006; 132:327-353.

10. Schaufeli WB. Past performance and future perspectives of burnout research. J Ind Psychol. 2003;29:1-15.

11. Maslach C. Job burnout: New directions in research and intervention. Curr Dir Psychol Sci. 2003; 12:189-192.

12. Lee RT, Ashforth BE. A meta-analytic examination of the correlates of the three dimensions of job burnout. J Appl Psychol; 1996; 81(2):123-133.

13. Maslach C. Different perspectives on job burnout. PsycCRITIQUES, 2004; 49(2):168-170.

14. Bakker AB, Demerouti $E$, Schaufeli WB. Validation of the Maslach Burnout Inventory-General Survey: An Internet study across occupations. Anxiety Stress Copin. 2002; 15(3):245-260.

15. Vinje HF, Mittelmark MB. Job engagement's paradoxical role in nurse burnout. Nurs Health Sci. 2007; 9(2):107-111.

16. Pines $\mathrm{AM}$, Yafe-Yanai 0 . Unconscious determinants of career choice and burnout: Theoretical model and counseling strategy. J Employment Couns. 2001; 38(4):170-184.

17. Vanheule S, Lievrouw A, Verhaeghe P. Burnout and intersubjectivity: A psychoanalytical study from a Lacanian perspective. Hum Relat. 2003; 56(3):321-339.

18. Leiter $M$, Maslach, C. Burnout and quality in a sped-up world. J Qual Particip. 2001;24(2):48-51.

19. Cooper CL, Dewe P, O'Driscoll MP. Organizational stress: A review and critique of theory, research, and applications. London: Sage; 2001.

20. Schaufeli WB, Greenglass ER. Introduction to special issue on burnout and health. Psychol Health. 2001;16(5):501-510.

21. Schaufeli WB, Enzmann D. The Burnout Companion to Study and Practice: A Critical Analysis. London: Taylor \&t Francis; 1998.

22. Toch H. Stress in policing. Washington, DC: American Psychologica Association; 2002.

23. Pines A, Aronson E. Career burnout. Causes and cures. Nueva York: Free Press; 1988.

24. lacovides A, Fountoulakis KN, Kaprinis St, Kaprinis, G. The relationship between job stress, burnout and clinical depression. J Affect Disorders. 2003; 75(3):209-221.

25. Middeldorp CM, Cath DC, Boomsma DI. A twin-family study of association between employment, burnout and anxious depression. J Affect Disorders, 2006; 90(2-3):163-169.

26. Pines $A$, Aronson $E$, Kafry D. Burnout: From tedium to personal growth. In: Cherniss C., editor. Staff burnout: Job stress in the human services. Nueva York: The Free Press; 1981.
27. Shirom A, Ezrachi J. On the discriminant validity of burnout, depression and anxiety. Anxiety Stress Copin. 2003;16(1):83-99.

28. Topa $G$, Morales JF. Determinantes específicos de la satisfacción laboral, el burnout y sus consecuencias para la salud: un estudio exploratorio con funcionarios de prisiones. Int J Psychol Psychol Ther. 2005; 5(1):71-81.

29. Moreno B, Peñacoba $C$. El estrés asistencial en los servicios de salud. En: Simón MA., editores. Manual de psicología de la salud. Fundamentos, metodología y aplicaciones. Madrid: Biblioteca Nueva; 1999.

30. Glass DC, McKnight JD. Perceived control, depressive symptomatology, and professional burnout: a review of the evidence. Psychol health. 1996;11(1):23-48.

31. Bakker AB, Schaufeli WB, Demerouti E, Janssen PMP, Van der Hulst $R$, Brouwer $J$. Using equity theory to examine the difference between burnout and depression. Anxiety Stress Copin. 2000;13(3):247-268.

32. Jiménez $\mathrm{C}$, Morales $\mathrm{JL}$, Martínez $\mathrm{C}$. Estudio del sindrome de "Burnout» en cirujanos pediatras españoles. Cir Pediatr. 2002:15(2):73-78.

33. Selye $H$. The stress of life. New York: McGraw Hill; 1956.

34. Hart PM, Cooper CL. Occupational stress: Toward a more integrated framework. In: Anderson N, Ones DS, Sinangil HK, Viswesvaran C., editors. Handbook of industrial, work and organizational psychology. Thousand Oaks, CA: Sage; 2001. p. 93-114.

35. Lazarus RS. Estrés y emoción. Manejo e implicaciones en nuestra salud. Bilbao: Desclée de Brouwer; 2000.

36. Halbesleben JR, Buckley MR. Burnout in Organizational Life. J Manage. 2004;30(6):859-879.

37. Evans BK, Fisher DG. The nature of burnout: a study of the three factor model of burnout in human service and non-human service samples. J Occup Organ Psych. 1993; 66(1):29-38.

38. Hillhouse J, Adler C, Waltwers D. A simple model of stress, burnout, and symptomatology in medical residents: A longitudinal study. Psychol Health Med. 2000; 5(1):63-73.

39. Maslach C, Jackson SE. The measurement of experienced burnout. J Organ Behav. 1981; 2(2):99-113.

40. Schaufeli W. Burnout. In: Firth-Cozens J, Payne RL, editores. Stress in health professionals. Psichological and organizational causes and interventions. Chichester: John Wiley; 1999. p.12-32.

41. Maslach C, Jackson SE. Maslach Burnout Inventory Manual. 2a ed. Palo Alto, CA: Consulting Psychologists Press; 1986.

42. Maslach $C_{1}$ Jackson SE, Leiter MP. Maslach Burnout Inventory Manual. 3a ed. Palo Alto, CA: Consulting Psychologists Press; 1996.

43. Golembiewski RT, Boudreau RA, Munzenrider RF, Lou H. Global burnout: A worldwide pandemic explored by the phase mode. Greenwich: JAI Press; 1996. Monographs in organizational behaviour and industrial relations; 21.

44. Aluja A, Blanch A, Garcia LF. Dimensionality of the Maslach Burnout Inventory in school teachers: a study of several proposals. Eur J Psychol Assess. 2005; 21(1):67-76.

45. Shirom A, Melamed S. Comparison of the construct validity of two burnout measures in two groups of professionals. Int J Stress Manage. 2006; 13(2):176-200

46. Schaufeli WB, Van Dierendonck D. The construct validity of two burnout measures. J Occup Behav. 1993; 14(7):631-47.

47. Boles JS, Dean DH, Ricks JM, Short JC, Wang G. The dimensionality of the Maslach Burnout Inventory across small business owners and educators. J Vocat Behav, 2000; 56(1):12-34

\section{Referencia complementaria}

Jimmieson N. Employee reactions to behavioural control under conditions of stress: The moderating role of self-efficacy. Work Stress. 2000;14(3):262-280. 